\title{
ASYMPTOTIC INTEGRATION OF A SECOND ORDER ORDINARY DIFFERENTIAL EQUATION
}

\author{
JAROMÍR ŠIMŠA
}

\begin{abstract}
Equation (1) $\left(r(t) x^{\prime}\right)^{\prime}+f(t) x=0$ is regarded as a perturbation of $(2)\left(r(t) y^{\prime}\right)^{\prime}+g(t) y=0$, where the latter is nonoscillatory at infinity. It is shown that if a certain improper integral involving $f-g$ converges sufficiently rapidly (but perhaps conditionally), then (1) has a solution which behaves for large $t$ like a principal solution of (2). The proof of this result is presented in such a way that it also yields as a by-product an improvement on a recent related result of Trench.
\end{abstract}

Let $f, g$, and $r$ be continuous on $[a, \infty)$. Consider the scalar differential equation

$$
\left(r(t) x^{\prime}\right)^{\prime}+f(t) x=0 \quad(r(t)>0, t \geq a)
$$

as a perturbation of

$$
\left(r(t) y^{\prime}\right)^{\prime}+g(t) y=0 .
$$

The quantities $x$ and $f$ in (1) may be complex-valued, while $g$ and $y$ in (2) are necessarily real-valued. Assume also that (2) is nonoscillatory at infinity. Then (2) has solutions $y_{1}$ and $y_{2}$ which are positive on $[b, \infty)$ for some $b \geq a$ and satisfy

$$
r\left(y_{1} y_{2}^{\prime}-y_{1}^{\prime} y_{2}\right)=1
$$

and the function

$$
\rho=y_{2} / y_{1}
$$

approaches infinity monotonically as $t \rightarrow \infty$. The solution $y_{1}$ is said to be a principal solution of (2) (see [1, p. 355]).

The following problem has already received much attention: What conditions on $f-g$ imply that (1) has a solution $x_{1}$ which behaves for large $t$ like $y_{1}$ ? It seems to be reasonable to assume that the improper integrals

$$
G_{1}(t)=\int_{t}^{\infty} y_{1} y_{2}(f-g) d s \quad \text { and } \quad G_{2}(t)=\int_{t}^{\infty} y_{1}^{2}(f-g) d s
$$

converge (perhaps conditionally). As noticed in [2], $G_{2}$ exists and satisfies

$$
\left|G_{2}(t)\right| \leq \frac{2}{\rho(t)} \sup _{s \geq t}\left|G_{1}(s)\right| \quad(t \geq b)
$$

provided that $G_{1}$ exists.

The following is our main result. Throughout the paper, $\varphi$ is positive and nonincreasing on $[a, \infty)$, with $\varphi(t) \rightarrow 0$ as $t \rightarrow \infty$. The symbol " $O$ " refers to behavior as $t \rightarrow \infty$.

Received by the editors May 27, 1986.

1980 Mathematics Subject Classification (1985 Revision). Primary 34C99. 
THEOREM 1. Suppose that $G_{1}$ exists and satisfies

$$
G_{1}=O(\varphi)
$$

If

(8)

$$
\int^{\infty}\left|G_{1}\right| \frac{\rho^{\prime}}{\rho} \varphi d t<\infty
$$

then (1) has a solution $x_{1}$ satisfying

$$
x_{1}=\left[1+O\left(\varphi_{1}\right)\right] y_{1}
$$

and

$$
\left(x_{1} / y_{1}\right)^{\prime}=O\left(\varphi \rho^{\prime} / \rho\right)
$$

where

$$
\varphi_{1}(t)=\max \left\{\varphi(t), \int_{t}^{\infty}\left|G_{1}\right| \frac{\rho^{\prime}}{\rho} \varphi d s\right\} .
$$

We take a more general approach in the proof to obtain a useful by-product (Theorem 2) which improves upon a recent result of Trench [2]. We present also an example showing that Theorem 1 is applicable where Theorem 2 is not.

THEOREM 2. Suppose that $G_{1}$ exists and satisfies (7). If

$$
\int^{\infty}\left|G_{2}\right| \rho^{\prime} \varphi d s<\infty
$$

and

$$
\varlimsup_{t \rightarrow \infty}(\varphi(t))^{-1} \int_{t}^{\infty}\left|G_{2}\right| \rho^{\prime} \varphi d s<1
$$

then (1) has a solution $x_{1}$ satisfying (10) and

$$
x_{1}=[1+O(\varphi)] y_{1} \text {. }
$$

Actually, the result of [2] is that Theorem 2 holds if the left side of (13) is smaller than $1 / 3$.

REMARK. By an argument given in [2], it is possible to choose constants $c$ and $b_{1}(\geq b)$ so that the function

$$
x_{2}(t)=x_{1}(t)\left[c+\int_{b_{1}}^{t} \frac{d s}{r x_{1}^{2}}\right]
$$

is a solution of (1) and behaves like $y_{2}$ as $t \rightarrow \infty$. We omit this discussion here.

We will combine the proofs of Theorems 1 and 2. It is convenient to define

$$
\left(\mathcal{M}_{1} z\right)(t)=\int_{t}^{\infty} y_{1} y_{2}(f-g) z d s \quad(t \geq b)
$$

and

$$
\left(\mathcal{M}_{2} z\right)(t)=\int_{t}^{\infty} y_{1}^{2}(f-g) z d s \quad(t \geq b)
$$

Following Trench [2], the solution $x_{1}$ of (1) will be found in the form

$$
x_{1}=\left(1+z_{1}\right) y_{1},
$$


where $z_{1}$ is the fixed point (function) of the mapping

$$
\tau z=G_{1}-\rho G_{2}+\mathcal{M}_{1} z-\rho \mathcal{M}_{2} z .
$$

We let $\tau$ act on the Banach space

$$
B=\left\{z \in C^{\prime}\left[t_{0}, \infty\right) \mid z=O\left(\varphi_{1}\right), z^{\prime}=O\left(\varphi \rho^{\prime} / \rho\right)\right\}
$$

with norm

$$
\|z\|=\sup _{t \geq t_{0}} \max \left\{\frac{|z|}{\varphi_{1}}, \frac{\left|z^{\prime}\right| \rho}{K \varphi \rho^{\prime}}\right\}
$$

where the function $\varphi_{1}$ and the constants $t_{0}$ and $K$ will be specified later. (Trench [2] considered the case $\varphi_{1}=\varphi$ and $K=2$.) Now we suppose only that $t \geq t_{0} \geq$ $b, K>0, \varphi_{1}(t) \geq \varphi(t)$, and $\varphi_{1} \rightarrow 0$ monotonically as $t \rightarrow \infty$. Finally, suppose that $G_{j}$ in (5) exist and satisfy

$$
\left|G_{1}(t)\right|+\rho(t)\left|G_{2}(t)\right| \leq \varphi(t)
$$

(see (6) and (7)), otherwise $\varphi$ is replaced by $c \varphi$, where $c$ is a sufficiently large constant. Then $\mathcal{M}_{2} z$ exists and satisifies

$$
\left|\left(\mathcal{M}_{2} z\right)(t)\right| \leq\|z\|\left[\varphi_{1}(t)+K \varphi(t)\right] \varphi(t) / \rho(t)
$$

for any $z \in B$. To see this, we first observe from (20) that

$$
|z| \leq\|z\| \varphi_{1} \text { and }\left|z^{\prime}\right| \leq K\|z\| \varphi \rho^{\prime} / \rho .
$$

Rewriting (16) and integrating by parts yields

$$
\left(\mathcal{M}_{2} z\right)(t)=-\int_{t}^{\infty} G_{2}^{\prime} z d s=G_{2}(t) z(t)+\int_{t}^{\infty} G_{2} z^{\prime} d s .
$$

In view of (21) and (23), this integration is valid, since $\left|G_{2} z\right| \leq\|z\| \varphi_{1} \varphi / \rho$, the integral on the right side converges absolutely, and is dominated by

$$
K\|z\| \varphi^{2}(t) \int_{t}^{\infty} \frac{\rho^{\prime}}{\rho^{2}} d s=\frac{K\|z\| \varphi^{2}(t)}{p(t)} .
$$

This implies (22). Similarly, using the equalities

$$
\left(\mathcal{M}_{1} z\right)(t)=G_{2}(t) \rho(t) z(t)+\int_{t}^{\infty} G_{2}(\rho z)^{\prime} d s
$$

and

$$
\left(\mathcal{M}_{1} z\right)(t)=G_{1}(t) z(t)+\int_{t}^{\infty} G_{1} z^{\prime} d s
$$

(see (4), (5), and (15)), it is easy to check that (21) and (23) imply the existence of $\mathcal{M}_{1} z$, with

$$
\left|\left(\mathcal{M}_{1} z\right)(t)\right| \leq\|z\|\left[\varphi_{1}(t) \varphi(t)+\int_{t}^{\infty}\left|G_{2}\right| \rho^{\prime}\left(\varphi_{1}+K \varphi\right) d s\right]
$$

or

$$
\left|\left(\mathcal{M}_{1} z\right)(t)\right| \leq\|z\|\left[\varphi_{1}(t) \varphi(t)+K \int_{t}^{\infty}\left|G_{1}\right| \frac{\rho^{\prime}}{\rho} \varphi d s\right]
$$

for any $z \in B$, provided that the corresponding right side is finite. 
Differentiating in (5), (15), and (16) yields

$$
\left(G_{1}-\rho G_{2}\right)^{\prime}=-\rho^{\prime} G_{2} \text { and }\left(\mathcal{M}_{1} z-\rho \mathcal{M}_{2} z\right)^{\prime}=-\rho^{\prime} \mathcal{M}_{2} z
$$

To prove Theorem 1, assume that (8) holds, and choose $\varphi_{1}$ as in (11). Then the right side of (25) is dominated by $\|z\|(K+\varphi) \varphi_{1}$. Consequently, (22), (25), and (26) imply that the function

$$
\mathcal{L} z=\mathcal{M}_{1} z-\rho \mathcal{M}_{2} z
$$

is continuously differentiable and satisfies

$$
\sup _{t \geq t_{0}} \frac{|\mathcal{L} z|}{\varphi_{1}} \leq\|z\|\left[2 \varphi\left(t_{0}\right)+K+K \varphi\left(t_{0}\right)\right]
$$

and

$$
\sup _{t \geq t_{0}} \frac{\left|(\mathcal{L} z)^{\prime}\right| \rho}{K \varphi \rho^{\prime}} \leq\|z\|\left[\varphi\left(t_{0}\right)+K^{-1} \varphi_{1}\left(t_{0}\right)\right] .
$$

Now we can choose $K=1 / 2$ and a large $t_{0}$ so that the right sides of (28) and (29) are dominated by $A\|z\|$, with a constant $A<1$. This and definition (20) imply that $\|\mathcal{L} z\| \leq A\|z\|$ for any $z \in B$. From (19), (21), and (26) we see that $\left(G_{1}-\rho G_{2}\right) \in B$. Thus $\tau$ in (18) is a contraction on $B$. If $z_{1}$ is the (unique) fixed point of $\tau$, then $x_{1}$ in (17) is a solution of (1) satisfying (9) and (10), because of $z_{1} \in B$. This completes the proof Theorem 1.

To prove Theorem 2 , we put $\varphi_{1}=\varphi$. If (12) holds, then (22), (24), and (26) imply that the function $\mathcal{L} z$ in (27) is continuously differentiable and satisfies

$$
\sup _{t \geq t_{0}} \frac{|\mathcal{L} z|}{\varphi} \leq\|z\|\left[(K+1) \sup _{t \geq t_{0}}(\varphi(t))^{-1} \int_{t}^{\infty}\left|G_{2}\right| \rho^{\prime} \varphi d s+(K+2) \varphi\left(t_{0}\right)\right]
$$

and

$$
\sup _{t \geq t_{0}} \frac{\left|(\mathcal{L} z)^{\prime}\right| \rho}{K \varphi \rho^{\prime}} \leq\|z\|\left(1+K^{-1}\right) \varphi\left(t_{0}\right)
$$

Assuming (13) holds, we now choose $K>0$ so that

$$
(K+1) \varlimsup_{t \rightarrow \infty}(\varphi(t))^{-1} \int_{t}^{\infty}\left|G_{2}\right| \rho^{\prime} \varphi d s<1 .
$$

Since $\varphi\left(t_{0}\right) \rightarrow 0$ as $t_{0} \rightarrow \infty$, there exists a $t_{0}$ so that the right sides of (30) and (31) are dominated by $A\|z\|$, with a constant $A<1$. The rest of the proof is the same as that of Theorem 1.

EXAMPLE. Trench [2] has considered the equation

$$
x^{\prime \prime}+K\left[t^{-1}(\log t)^{-\alpha} \sin t\right] x=0,
$$

where $K$ and $\alpha$ are nonzero constants, as a perturbation of $y^{\prime \prime}=0$. Then $y_{1}=$ $1, y_{2}=\rho=t$, and

$$
\begin{aligned}
G_{1}(t) & =K \int_{t}^{\infty}(\log s)^{-\alpha} \sin s d s \\
& =\left[K \cos t+O\left(t^{-1}\right)\right](\log t)^{-\alpha},
\end{aligned}
$$


provided that $\alpha>0$ (see (5) and use integration by parts). Thus (7) holds with $\varphi=(\log t)^{-\alpha}$. For this $\varphi$ and $\alpha>1 / 2$,

$$
\int_{t}^{\infty} \frac{\rho^{\prime}}{\rho} \varphi^{2} d s=\int_{t}^{\infty} \frac{(\log s)^{-2 \alpha}}{s} d s=\frac{(\log t)^{1-2 \alpha}}{2 \alpha-1}
$$

which proves (8) because (7). Theorem 1 implies that (32) has a solution $x_{1}$ satisfying

$$
x_{1}(t)=1+O\left((\log t)^{-\beta}\right) \quad \text { and } \quad x_{1}^{\prime}(t)=O\left(t^{-1}(\log t)^{-\alpha}\right)
$$

with $\beta=\min \{\alpha, 2 \alpha-1\}$, provided that $\alpha>1 / 2$. As shown in [2], the left side of (13) is not finite here unless $\alpha \geq 1$. So Theorem 2 does not apply if $\alpha<1$.

ACKNOWLEDGMENT. I wish to thank to Professor Trench for a preprint of [2] and for many helpful suggestions on the present work.

\section{REFERENCES}

1. P. Hartman, Ordinary differential equations, Wiley, New York, 1964.

2. W. F. Trench, Linear perturbations of a nonoscillatory second order equation, Proc. Amer. Math. Soc. 97 (1986), 423-428.

Department of Mathematics, J. E. Purkyne University, Janackovo nam. 2A, 66295 BRNO, CZECHOSLOVAKIA 